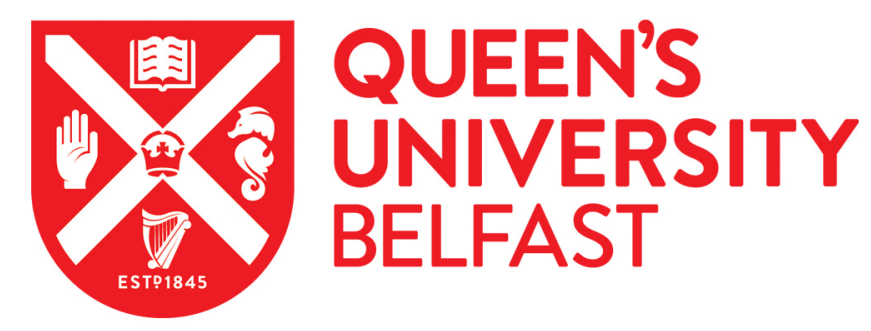

\title{
Clouds on the Hot Jupiter HD189733b: Constraints from the Reflection Spectrum
}

Barstow, J. K., Aigrain, S., Irwin, P. G. J., Hackler, T., Fletcher, L. N., Lee, J. M., \& Gibson, N. P. (2014). Clouds on the Hot Jupiter HD189733b: Constraints from the Reflection Spectrum. The Astrophysical Journal, 786(2). https://doi.org/10.1088/0004-637X/786/2/154

\section{Published in:}

The Astrophysical Journal

Document Version:

Publisher's PDF, also known as Version of record

Queen's University Belfast - Research Portal:

Link to publication record in Queen's University Belfast Research Portal

Publisher rights

Copyright 2014 The American Astrophysical Society.

This work is made available online in accordance with the publisher's policies. Please refer to any applicable terms of use of the publisher.

\section{General rights}

Copyright for the publications made accessible via the Queen's University Belfast Research Portal is retained by the author(s) and / or other copyright owners and it is a condition of accessing these publications that users recognise and abide by the legal requirements associated with these rights.

Take down policy

The Research Portal is Queen's institutional repository that provides access to Queen's research output. Every effort has been made to ensure that content in the Research Portal does not infringe any person's rights, or applicable UK laws. If you discover content in the Research Portal that you believe breaches copyright or violates any law, please contact openaccess@qub.ac.uk. 


\title{
CLOUDS ON THE HOT JUPITER HD189733b: CONSTRAINTS FROM THE REFLECTION SPECTRUM
}

\author{
J. K. Barstow ${ }^{1}$, S. Aigrain ${ }^{1}$, P. G. J. Irwin ${ }^{1}$, T. Hackler ${ }^{1}$, L. N. Fletcher ${ }^{1}$, J. M. LeE ${ }^{2}$, And N. P. Gibson ${ }^{3}$ \\ ${ }^{1}$ Department of Physics, University of Oxford, Oxford, UK; jo.barstow@ astro.ox.ac.uk \\ ${ }^{2}$ Institute for Theoretical Physics, University of Zürich, CH-8057 Zürich, Switzerland \\ ${ }^{3}$ European Southern Observatory, D-85748 Garching bei München, Germany \\ Received 2013 December 3; accepted 2014 March 25; published 2014 April 25
}

\begin{abstract}
The hot Jupiter HD 189733b is probably the best studied of the known extrasolar planets, with published transit and eclipse spectra covering the near UV to mid-IR range. Recent work on the transmission spectrum has shown clear evidence for the presence of clouds in its atmosphere, which significantly increases the model atmosphere parameter space that must be explored in order to fully characterize this planet. In this work, we apply the NEMESIS atmospheric retrieval code to the recently published HST/STIS reflection spectrum, and also to the dayside thermal emission spectrum in light of new Spitzer/IRAC measurements, as well as our own re-analysis of the HST/NICMOS data. We first use the STIS data to place some constraints on the nature of clouds on HD 189733b and explore solution degeneracy between different cloud properties and the abundance of $\mathrm{Na}$ in the atmosphere; as already noted in previous work, absorption due to Na plays a significant role in determining the shape of the reflection spectrum. We then perform a new retrieval of the temperature profile and abundances of $\mathrm{H}_{2} \mathrm{O}, \mathrm{CO}_{2}, \mathrm{CO}$, and $\mathrm{CH}_{4}$ from the dayside thermal emission spectrum. Finally, we investigate the effect of including cloud in the model on this retrieval process. We find that the current quality of data does not warrant the extra complexity introduced by including cloud in the model; however, future data are likely to be of sufficient resolution and signal-to-noise that a more complete model, including scattering particles, will be required.
\end{abstract}

Key words: methods: data analysis - planets and satellites: atmospheres - radiative transfer

Online-only material: color figures

\section{INTRODUCTION}

Since its discovery in 2005 (Bouchy et al. 2005), the hot Jupiter HD 189733b has been repeatedly observed as it transits and is eclipsed by its parent star, leading to excellent coverage in both its transmission and eclipse spectra from the visible to mid-infrared. This has resulted in HD 189733b being probably the best characterized of all the known exoplanets; it is known from the observed slope of the reflectance spectrum that this unresolved planet would appear a deep shade of blue (Evans et al. 2013), a fact that testifies to the power of the transit spectroscopy technique. The transmission spectrum presented by Pont et al. (2013) shows clear evidence for haze or cloud in the atmosphere of this planet, making it the first transiting planet outside the solar system that is known to be cloudy. Lee et al. (2012) and Line et al. (2012) analyze the dayside spectrum from secondary eclipse observations, and retrieve the temperature-pressure profile and abundances of $\mathrm{H}_{2} \mathrm{O}, \mathrm{CO}_{2}, \mathrm{CO}$, and $\mathrm{CH}_{4}$. Knutson et al. (2012) use Spitzer/IRAC phase curves to investigate the longitudinal temperature variability, and de Wit et al. (2012) combine the phase curves with an analysis of the ingress/egress shape in eclipse to place further constraint on spatial variability.

The recent albedo spectrum from $H S T$ /STIS obtained by Evans et al. (2013) provides an opportunity to investigate the cloud structure on the dayside. Unlike the transmission spectrum investigated by Pont et al. (2013) and Lee et al. (2013b) which probes the limb of the exoplanet atmosphere, the dayside reflection spectrum has near-nadir geometry, and so it is sensitive to deeper regions of the atmosphere. If the cloud is similar at the terminators and on the dayside, we can use this to further constrain its properties. Alternatively, we may see an entirely different cloud layer on the hotter dayside from that observed at the terminator. The albedo spectrum is useful for placing constraints on the cloud, as scattering particles have a significant effect on the optical reflectivity of an atmosphere. We expect there to be fewer gaseous absorbing species in the visible part of the spectrum than in the infrared; distinct absorption features of $\mathrm{Na}$ and $\mathrm{K}$ are seen in the transmission spectrum, but otherwise cloud appears to be the dominant opacity source in this region (Pont et al. 2013). We also expect little, if any, thermal contribution from the planet itself at these wavelengths due to its temperature.

In this work, we use the Non-linear optimal Estimator for MultivariatE spectral analySIS (NEMESIS) software (Irwin et al. 2008) to calculate synthetic spectra using a simple cloudy model atmosphere for HD 189733b, including multiple scattering. We vary the cloud parameters and the Na volume mixing ratio (VMR) and compare the resultant spectra to the STIS measurement; calculating the $\chi^{2}$ goodness-of-fit parameter allows us to determine the region of model parameter space that provides the best match. We then use a subset of our best-fitting models to examine the effect of clouds on our ability to accurately retrieve temperature and molecular abundances from the infrared dayside emission spectrum, following the work of Lee et al. (2012).

\subsection{NEMESIS}

We use the NEMESIS spectral retrieval tool to produce forward models (predicted spectra for a range of model atmospheres) for comparison with the HST/STIS spectra, and also to retrieve the atmospheric state from the thermal emission spectrum as in Lee et al. (2012). NEMESIS was developed by Irwin et al. (2008) for atmospheric retrieval of solar system planets, and it has since been extended to enable the same analysis for observations of transiting and directly imaged extrasolar planets 
Table 1

Sources of Gas Absorption Line Data

\begin{tabular}{lc}
\hline \hline $\mathrm{Gas}$ & Source \\
\hline $\mathrm{H}_{2} \mathrm{O}$ & HITEMP2010 (Rothman et al. 2010) \\
$\mathrm{CO}_{2}$ & CDSD-1000 (Tashkun et al. 2003) \\
$\mathrm{CO}$ & HITRAN1995 (Rothman et al. 1995) \\
$\mathrm{CH}_{4}$ & STDS (Wenger \& Champion 1998) \\
$\mathrm{Na}$ & VALD (Heiter et al. 2008) \\
$\mathrm{K}$ & VALD (Heiter et al. 2008) \\
\hline
\end{tabular}

(Lee et al. 2012, 2013a; Barstow et al. 2013a). NEMESIS is an Optimal Estimation retrieval model (Rodgers 2000) and uses a correlated- $k$ radiative transfer model (Lacis \& Oinas 1991; Goody \& Yung 1989). NEMESIS is not a radiative equilibrium model; instead, it simply uses the atmospheric model provided to compute the incoming and outgoing radiative flux. In an irradiated case, it will compute the incoming and scattered/ reflected flux from the star, but it will not take into account the heating effect of the incoming stellar flux on the atmosphere. For the multiple scattering runs, NEMESIS uses beams over a user-specified number of zenith angles; azimuthal dependence is accounted for using Fourier decomposition. For more details of the scattering calculation in this work, see Section 1.1.1.

We use the same line and collision-induced absorption data as Lee et al. (2012), Lee et al. (2013b), and Barstow et al. (2013b). A list of sources for absorption line data is given in Table $1 . \mathrm{H}_{2}-\mathrm{He}$ collision-induced absorption data are taken from the models of Borysow \& Frommhold (1989), Borysow et al. (1989), Borysow \& Frommhold (1990), Borysow et al. (1997), and Borysow (2002). The reference stellar spectrum is taken from the model set made available by Kurucz, ${ }^{4}$ and the stellar radius is taken from Baines et al. (2008). We use the same planetary mass and radius as Lee et al. (2013b), and a $\mathrm{H}_{2}: \mathrm{He}$ ratio of 9:1. As found by Lee et al. (2012), the precise value of this does not have a large effect on secondary eclipse retrievals.

\subsubsection{Multiple Scattering Calculations}

In order to reproduce an accurate reflection spectrum in the presence of an optically thick cloud, it is necessary to include multiple scattering calculations as many scattering events are likely. NEMESIS uses the matrix operator algorithm of Plass et al. (1973) for multiple scattering calculations, where the zenith angle integration is achieved using a five-point Gaussian-Lobatto quadrature scheme and the azimuth angle integration is achieved through Fourier decomposition, with the necessary number of Fourier components determined by the stellar and emission zenith angles. The analytical disk-averaged integration scheme used in Lee et al. (2012) for eclipse spectra is not used here as it is not applicable to scattering situations; instead, we represent the disk average for the synthetic STIS spectra by running multiple scattering calculations with the stellar zenith angles set to each of the five Gaussian-Lobatto quadrature angles and the azimuth angle set for back-scattering, since during secondary transit the observer is located in the same direction as the star and the stellar zenith angle is equal to the emission angle. The disk-average is then determined using a weighted average of these five different calculations, assuming that the atmospheric conditions are the same at all points on the disk. Retrievals including multiple scattering are computationally expensive. Therefore, we anticipate that

\footnotetext{
4 http://kurucz.harvard.edu/stars/HD189733/
}

the majority of the thermal emission retrieval calculations in the future will still be performed with an extinction-only approximation using the disk integration described by Lee et al. (2012). However, we also tested the effect of including multiple scattering to test the sensitivity of the retrieval to differences in our modeling approach. The five-angle approach used to calculate the STIS synthetic spectra is still too timeconsuming for the emission spectrum retrieval, so in this case we approximate further by calculating the spectrum for a stellar zenith angle of $45^{\circ}$ only, which represents the average angle of the weighting function that was used to compute the diskaveraged spectrum since

$$
\bar{R}=\int_{0}^{\pi / 2} 2 R(\theta) \sin (\theta) \cos (\theta) d \theta=\int_{0}^{\pi / 2} R(\theta) \sin (2 \theta) d \theta .
$$

The scattering phase function of the particles is calculated using Mie theory and approximated by the Henyey-Greenstein parameterization (Henyey \& Greenstein 1941), as in previous work on planetary clouds (e.g., Irwin et al. 2009; Barstow et al. 2012); expected deviations from the true phase function are small compared with the errors on the observed spectrum, so we consider this approximation to be valid. We use the doublepeaked version of the phase function:

$$
\begin{aligned}
P(\theta)= & \frac{1}{4 \pi}\left[f \frac{1-g_{1}^{2}}{\left(1+g_{1}^{2}-2 g_{1} \mu\right)^{3 / 2}}\right. \\
& \left.+(1-f) \frac{1-g_{2}^{2}}{\left(1+g_{2}^{2}-2 g_{2} \mu\right)^{3 / 2}}\right],
\end{aligned}
$$

which represents the phase function as the sum of forward and backward scattering peaks. Here, $\mu$ is the cosine of the scattering angle; $g_{1}$ and $g_{2}$ are scattering asymmetry parameters for the forward and backward peaks, respectively; and $f$ is the fractional contribution of the forward peak to the total phase function. For smaller particles approaching the Rayleigh scattering limit, the parameter $f$ is close to 0.5 and there are approximately equal contributions from the forward and backward scattering peaks; as the particle size increases relative to the wavelength of light the value of $f$ increases and the scattering becomes more asymmetric. However, in no case is the scattering completely isotropic.

We use the enstatite refractive index values of Scott \& Duley (1996) and the MnS values from Huffman \& Wild (1967) in our calculations of the scattering parameters. It is worth noting that, as we model realistic particles, they absorb as well as scatter incident radiation. The fraction of light that is absorbed rather than scattered is dependent on the particle size and also on the composition of the particles. In general, more of these realistic particles would be required than idealized, perfectly scattering particles to produce an equivalent planetary albedo in an atmospheric model, since for realistic particles some of the incident radiation is absorbed by the particles rather than scattered back to space. The single scattering albedo is also wavelength dependent, and this can therefore affect the shape of modeled planetary albedo and thermal emission spectra.

\section{DATA}

The HST/STIS data used in this work are presented in Evans et al. (2013). We use the tabulated eclipse depths binned in six channels, spanning the wavelength range $290-570 \mathrm{~nm}$, to place constraint on the cloud properties for HD 189733b. 


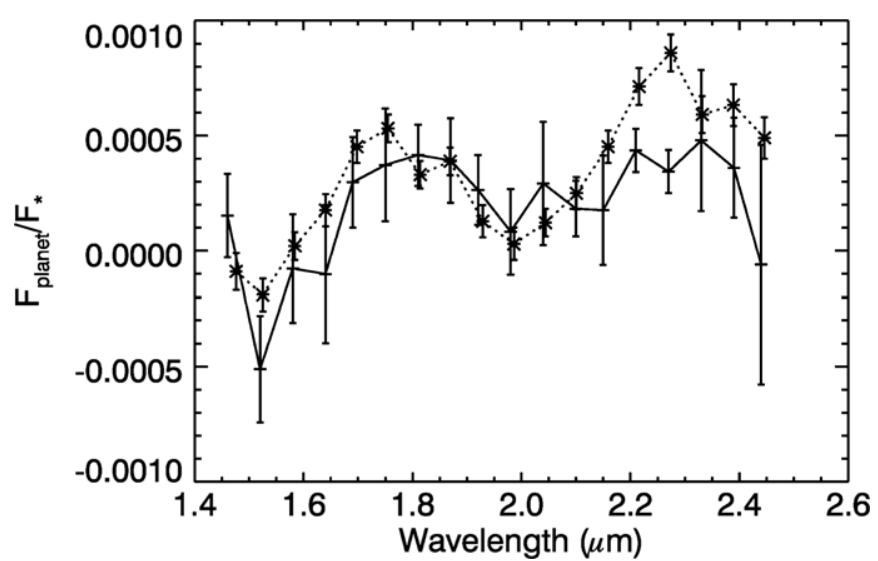

Figure 1. NICMOS spectrum as analyzed by Swain et al. (2009; stars, dotted line) and in this work (crosses, solid line). We find that the spectrum has a similar shape; but the feature amplitude is decreased, and the error bars are increased.

We also investigate the impact of including clouds on retrievals of temperature structure and atmospheric composition from the available thermal emission spectra. We use the same data as Lee et al. (2012), with the exception that we include the new values for the Spitzer/IRAC 3.6 and $4.5 \mu \mathrm{m}$ points presented in Knutson et al. (2012). Data used as in Lee et al. (2012) are the Spitzer/IRS spectrum from Grillmair et al. (2008), the Spitzer/IRS broadband point originally measured by Deming et al. (2006), and the broadband Spitzer MIPS and Spitzer/ IRAC 5.8/8.0 $\mu \mathrm{m}$ points from Charbonneau et al. (2008).

We also include a reanalysis of the HST/NICMOS observations of Swain et al. (2009). Gibson et al. (2011) re-analyzed a number of exoplanet transmission spectra obtained with NICMOS, including that of XO-1b, originally published by Tinetti et al. (2010). This re-analysis showed that the shape of the NICMOS spectra is highly sensitive to the assumptions made about the instrumental systematics, and that the errors may have been underestimated in the original publications. This was recently confirmed by new observations of $\mathrm{XO}-1 \mathrm{~b}$ in transit with HST/WFC3 (Deming 2013), which are less affected by systematics, and showed significant discrepancies with the original NICMOS data set. We expect that the NICMOS emission spectrum of HD189733b may suffer from the same problems as the aforementioned transmission spectra, but it is the only data set published to date in the crucial near-infrared wavelength range. We therefore re-analyzed it using a method proposed by Gibson et al. (2012), which makes minimal assumptions about the nature of the systematics. This re-analysis, which we describe briefly in Section 2.1, leads to larger but somewhat more robust error bars and thus to more conservative results for the retrieval analysis.

We omit the ground-based $K$ - and $L$-band data of Waldmann et al. (2012), as these data contain an extremely strong emission feature at $3.3 \mu \mathrm{m}$, which is attributed to non-local thermodynamic equilibrium processes; modeling this feature is therefore beyond the limits of our code. The attribution of this feature as resulting from the atmosphere of HD $189733 \mathrm{~b}$ has also been disputed by Mandell et al. (2011) as these authors did not detect it; they suggest it may be a result of telluric absorption. This conflict is so far unresolved, and as a result, we do not include these data in our analysis.

\subsection{Reanalysis of NICMOS Emission Spectrum}

We re-analyzed the NICMOS emission spectrum presented in Swain et al. (2009) using the method presented by Gibson
Table 2

The Reanalyzed NICMOS Spectrum

\begin{tabular}{lcl}
\hline \hline Wavelength & $\begin{array}{c}\text { Flux Ratio } \\
\left(10^{-4}\right)\end{array}$ & Error \\
\hline 1.4638 & 1.53 & 1.81 \\
1.5214 & -5.11 & 2.31 \\
1.5790 & -0.762 & 2.34 \\
1.6366 & -1.01 & 2.99 \\
1.6941 & 2.98 & 1.96 \\
1.7518 & 3.73 & 2.44 \\
1.8094 & 4.16 & 1.33 \\
1.8670 & 3.93 & 1.84 \\
1.9246 & 2.65 & 1.52 \\
1.9822 & 0.834 & 1.86 \\
2.0398 & 2.93 & 2.68 \\
2.0974 & 1.83 & 1.20 \\
2.1550 & 1.76 & 2.38 \\
2.2126 & 4.37 & 0.941 \\
2.2702 & 3.44 & 0.937 \\
2.3278 & 4.79 & 3.06 \\
2.3854 & 3.61 & 2.17 \\
2.4430 & -0.588 & 5.20 \\
\hline
\end{tabular}

et al. (2012). We briefly outline the process here, but we refer the interested reader to Gibson et al. (2012) for further information on the data reduction and the details of the systematics modeling method. We model the secondary eclipse and systematics simultaneously in each of 18 wavelength channels, using Gaussian Processes (GP) to model the dependence of the systematics on the instrumental parameters that are known to affect them: the orbital phase of HST $\phi$ and its square $\phi^{2}$; the $x$ and $y$-position of the spectral trace on the detector; and its width $w$ and its angle $\theta$ relative to the $x$-axis. The ephemeris, planet-to-star radius ratio, inclination, and system scale $\left(a / R_{\star}\right)$ were fixed to the values obtained by Winn et al. (2006). These are also consistent with the more recent determination of these parameters by Southworth (2008). The eccentricity was assumed to be zero, as there is no evidence in the literature for a non-zero eccentricity. We used three Markov-Chain Monte Carlo chains of 40,000 steps each to marginalize over all the parameters except the eclipse depth in each channel.

Figure 1 shows the spectrum obtained after re-analysis, with the original from Swain et al. (2009) for comparison. The two versions of the spectrum are largely similar in shape, but our error bars are about a factor of three larger, which is consistent with the findings of Gibson et al. (2012). The wavelength range probed by NICMOS is particularly sensitive to the gaseous abundances on the dayside of HD 189733b (Lee et al. 2012), so the increased error bars will impact the constraints we can extract from the thermal emission spectrum. The spectrum and $1 \sigma$ error bars are provided in numeric form in Table 2.

We note that the choice of which instrumental parameters to include in the systematics model for NICMOS affects both the shape of the resultant spectrum and the errors, whether using traditional linear de-correlation methods (Gibson et al. 2011) or our GP approach. However, in a GP model, the length scale $\eta$ associated with each parameter gives an indication of the relevance of that parameter (see Gibson et al. 2012, for details). In the present case, the best-fit length scales for all the instrument parameters we tried including in the model were found to be of the same order of magnitude, which is why we included them all in the final model. Nonetheless, this highlights the fact that the NICMOS results should be viewed with caution, as the spectrum is extremely sensitive to the details of the systematics removal. 
Table 3

The Values used for each Parameter in the Cloud Model, and for the Na Volume Mixing Ratio

\begin{tabular}{lc}
\hline \hline Variable & Values \\
\hline Base pressure $(\mathrm{mbar})$ & $1000,100,10,1$, uniform \\
Particle size $(\mu \mathrm{m})$ & $0.01,0.03,0.1,0.3,1,3,10$ \\
Optical depth at $0.25 \mu \mathrm{m}$ & $0.1,0.2,0.4,0.6,0.8,1,10$ \\
Na VMR (ppmv) & $0.5,5,50,500$ \\
\hline
\end{tabular}

Notes. A synthetic spectrum is generated for every combination of these parameters, making a total of 980 models. All cloud models span a decade in pressure, except for the uniform case-so if the base pressure is $1000 \mathrm{mbar}$, the top pressure is 100 mbar.

\section{CLOUD MODELS}

We use the HST/STIS data to investigate the range of cloud models that provide a reasonable match with HD 189733b's reflection spectrum. We use the best-fit atmospheric temperature profile retrieved by Lee (2012); the other parameters with a significant effect in the STIS wavelength range are all related to clouds, except for the abundance of sodium. Sodium has been detected in transmission spectra of HD 189733b (Redfield et al. 2008; Huitson et al. 2012), but it is not possible to obtain an absolute constraint on the sodium abundance from these measurements. Atmospheric models by Sudarsky et al. (2000) and Heng \& Demory (2013) suggest that Na should have a measurable effect on the reflection spectrum. The abundance is left as a free parameter in the model.

Clouds are extremely complex phenomena in radiative transfer. The resultant spectrum for a cloudy atmosphere depends on the scattering properties of the cloud, which in turn are affected by the composition and size of the cloud particles. The altitude and optical depth of the cloud affect how far light from the star will penetrate the atmosphere, which affects the fraction of the light that is absorbed before it can be reflected back. In order to investigate the potential cloud structure in the simplest possible way, the cloud has been modeled for a fixed set of particle sizes, optical depths, and cloud altitudes; the free parameters in our model for the STIS spectrum are listed in Table 3, with the range of values taken by each. The scattering efficiency and phase function for the cloud are calculated using Mie theory with a double-peaked Henyey-Greenstein approximation to the phase function; the scattering properties are therefore a consequence of the size and composition of the cloud particles.

All other parameters, including the atmospheric temperature and $\mathrm{H}_{2} \mathrm{O}, \mathrm{CO}_{2}, \mathrm{CO}$, and $\mathrm{CH}_{4}$ VMRs, are fixed during this analysis at the best-fit values of Lee (2012); none of these gases are expected to have strong absorption features between 0.3 and 0.6 , so their only effect on the spectrum is a small effect on the mean molecular weight. We also include Rayleigh scattering from $\mathrm{H}_{2}$ and $\mathrm{He}$, and the abundances of these gases are also fixed.

For most of the tests described below, we assume that the cloud particles are made of enstatite, $\mathrm{MgSiO}_{3}$ (Lecavelier Des Etangs et al. 2008; the refractive indices that were used are taken from Scott \& Duley 1996). This is likely to be condensed at the stratospheric temperature on HD 189733b (Fortney et al. 2010), but it is not the only chemical species that may be relevant; Morley et al. (2012) suggest that constituents such as MnS may also form clouds on hot Jupiters, and we consider the impact of changing the composition of the cloud particles in Section 3.2. Conversely, we do not expect to see absorption due to gaseous

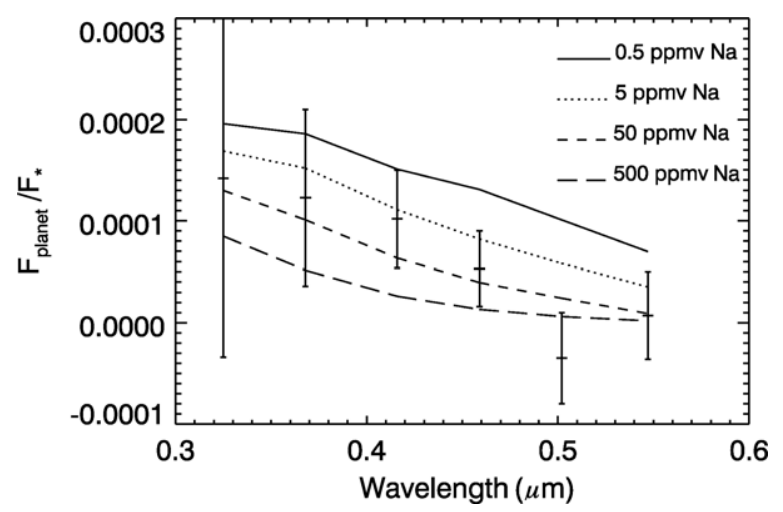

Figure 2. Six-channel STIS spectrum from Evans et al. (2013), with four cloudfree models overlaid. Models with $50 \mathrm{ppmv} \mathrm{Na}$ produce the best fit to the observed spectrum without the requirement for cloud; models with $0.5 \mathrm{ppmv}$ $\mathrm{Na}$ give a poor fit. $5 \mathrm{ppmv}$ is approximately solar abundance.

metal oxides such as $\mathrm{TiO}$ in the atmosphere as $\mathrm{Ti}$ is likely to be in condensate form at the expected photospheric temperature of HD 189733b (Fortney et al. 2010). This may mean that any condensates present also contain Ti oxides, as suggested by Helling \& Woitke (2006).

To test the effect of particle size, we use a range of monodisperse (single-size) cloud populations from $10 \mathrm{~nm}$ to $10 \mu \mathrm{m}$ in radius. For the STIS wavelength range, the Rayleigh scattering limit is approached for a particle size of $10 \mathrm{~nm}$, so the scattering behavior as a function of wavelength will be similar for any particles smaller than $10 \mathrm{~nm}$; the only difference would be that a larger number of smaller particles would be required to achieve the same optical depth. The nadir optical depths (referenced at $0.25 \mu \mathrm{m}$ ) are varied between 0.1 and 10 , and the cloud is located in a layer 1 decade thick in pressure coordinates (i.e., between 1000 and 100 mbar, or 100 and 10 mbar, etc.). We locate the cloud base at 1000,100,10, and $1 \mathrm{mbar}$ and also test the effect of distributing the cloud particles uniformly throughout the atmosphere. The cloud particle number density scale height is assumed to be the same as the pressure scale height in all cases.

Synthetic reflection spectra are generated for all combinations of these three cloud parameters and the $\mathrm{Na}$ abundance parameter, using NEMESIS. We list the values used for each parameter in Table 3.

We also generate a series of cloud-free models to investigate the impact of changing the sodium abundance by itself. It is clear that for a range of sodium abundances the STIS spectrum can be reproduced without the need for clouds (Figure 2; the effect of sodium on the spectrum is as predicted by Sudarsky et al. 2000), but if the atmosphere in fact contains more/ less sodium, additional scatterers/absorbers would be required (Heng \& Demory 2013).

For each of the cloudy models, the $\chi^{2}$ goodness-of-fit parameter is computed to indicate the quality of the fit to the 6 data points in the STIS spectrum. We use this as a means of comparing models across our parameter space; our 4 variables are Na VMR, cloud particle radius, cloud altitude, and cloud optical depth. For a good fit, therefore, the $\chi^{2}$ should approach $2\left(N_{\text {data }}-N_{\text {variables }}\right)$. Contour plots of the $\chi^{2}$ are shown for each parameter combination in Figure 3 , as well as the number of models for each value of each single parameter with a $\chi^{2}$ of less than 10 and less than 5 . The contour plots show two-dimensional cuts through the four-dimensional parameter space, with two parameters varying in each plot and the other two held fixed. 


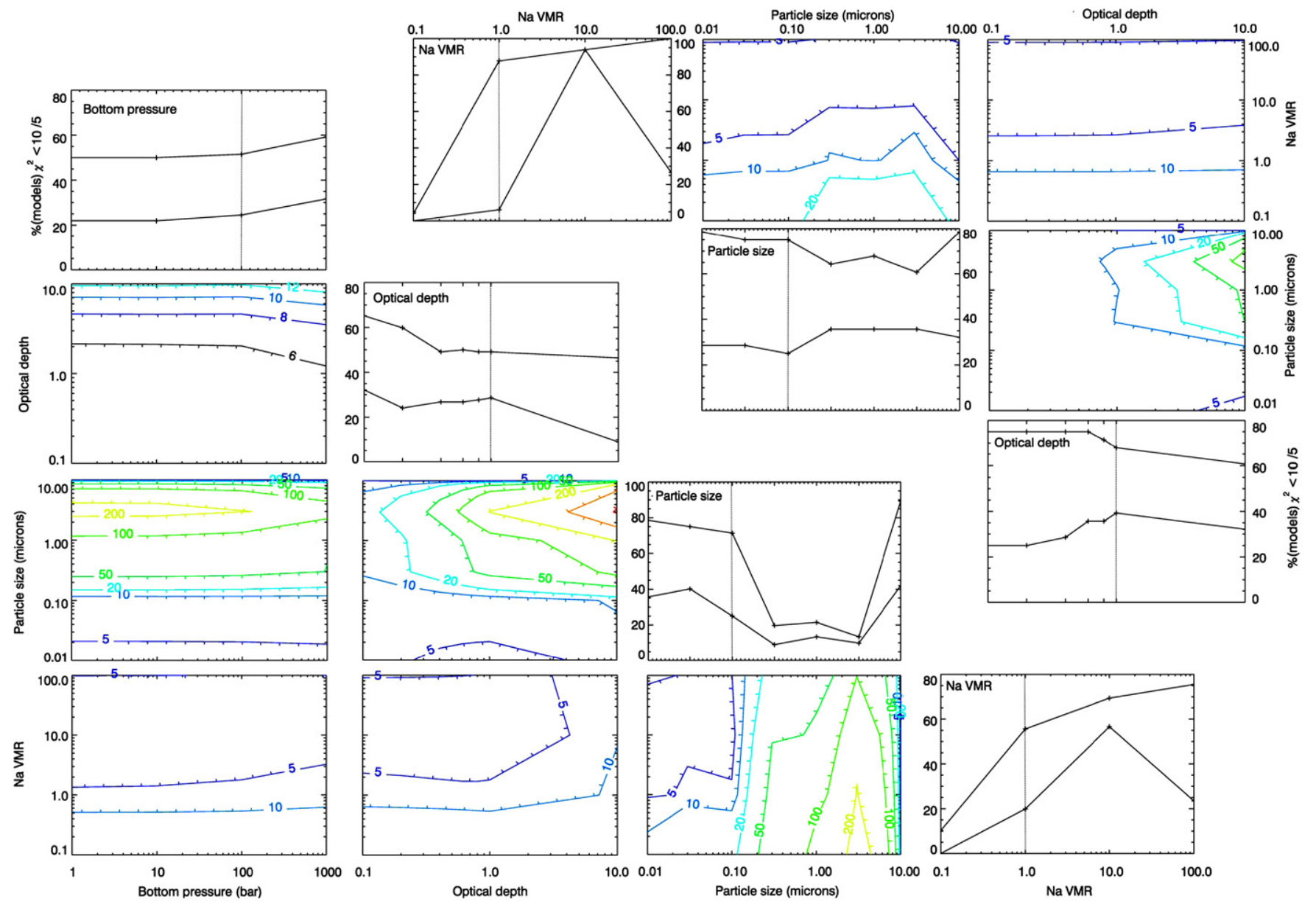

Figure 3. Contour plots of the $\chi^{2}$ goodness-of-fit parameter for (bottom left): cloud bottom pressure, optical depth, particle size, and Na volume mixing ratio; (top right): cloud optical depth, particle size, and $\mathrm{Na}$ volume mixing ratio for a uniformly mixed cloud. The Na VMR is quoted as a multiplication of the solar value $\left(\sim 5 \mathrm{ppmv}\right.$ ). Also plotted are the percentages of models with $\chi^{2}$ of $<10$ (top line) and $<5$ (bottom line) for each parameter. Each contour plot represents a $2 \mathrm{D}$ cut through the $4 \mathrm{D}$ parameter space, with two parameters varied and the other two held fixed in each plot. The dashed lines indicate the values used for each parameter when held fixed.

(A color version of this figure is available in the online journal.)

The values for each fixed parameter are indicated by the dashed lines in the histogram plots. This indicates that the best-fit region of parameter space occurs mostly for either small particles $(<0.3 \mu \mathrm{m}$ in size) or $10 \mu \mathrm{m}$ particles, $10 \times$ solar $\mathrm{Na}(50 \mathrm{ppmv})$, and optical depths of 1 or less. The preference for small particles $(0.01-0.1 \mu \mathrm{m})$ is consistent with the findings of Lecavelier Des Etangs et al. (2008).

A subsolar sodium abundance $(0.1 \times$, or $0.5 \mathrm{ppmv})$ is insufficient to reproduce the spectrum for the cases we test here. Examples of models with different cloud properties and $\mathrm{Na}$ VMRs are shown in Figure 4; models with 5-500 ppmv Na could all produce an adequate fit to the spectrum depending on the assumed cloud properties, but a model with $0.5 \mathrm{ppmv} \mathrm{Na}$ does not produce a sufficiently large absorption feature at wavelengths $>0.5 \mu \mathrm{m}$. In general, the cloud altitude is the model parameter with the smallest effect, except for the case where the sodium abundance is $100 \times$ solar (Figure 5). The degeneracy between sodium abundance and the cloud properties becomes complex when the sodium abundance is high, because the cloud must make the atmosphere optically thick at precisely the right altitude in order for the model to fit. For 50 ppmv sodium, a cloud-free model can fit the spectrum with a $\chi^{2}$ of less than 5 (Figure 2), but the increase in sodium abundance means that an

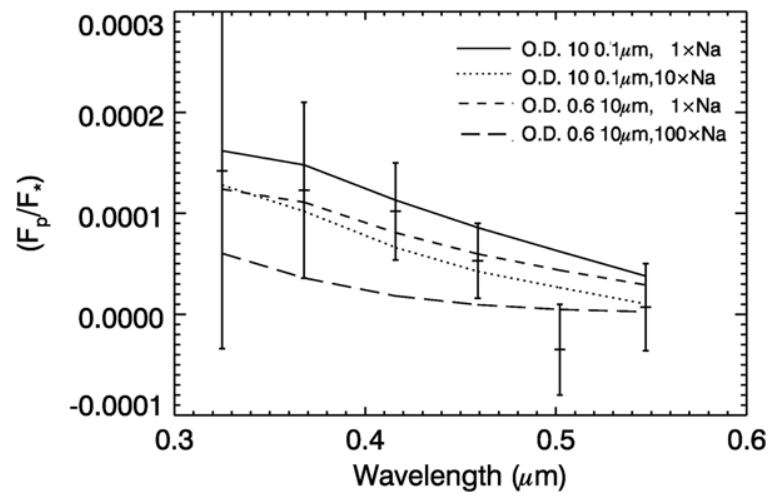

Figure 4. Spectra for a selection of cloud models with different properties and Na VMRs. The $0.1 \mu \mathrm{m}$ models are uniformly distributed in altitude, and the $10 \mu$ m models are between 1 and 0.1 mbar. The $0.1 \mu$ m model with $10 \times \mathrm{Na}$ and the $10 \mu$ m model with $1 \times \mathrm{Na}$ fit the spectrum well $\left(\chi^{2}<5\right)$, whereas the other two models do not.

additional reflective component is required in the model atmosphere for 500 ppmv.

The data do not allow us to distinguish between the finite deck-type models, where the cloud is confined to a certain 

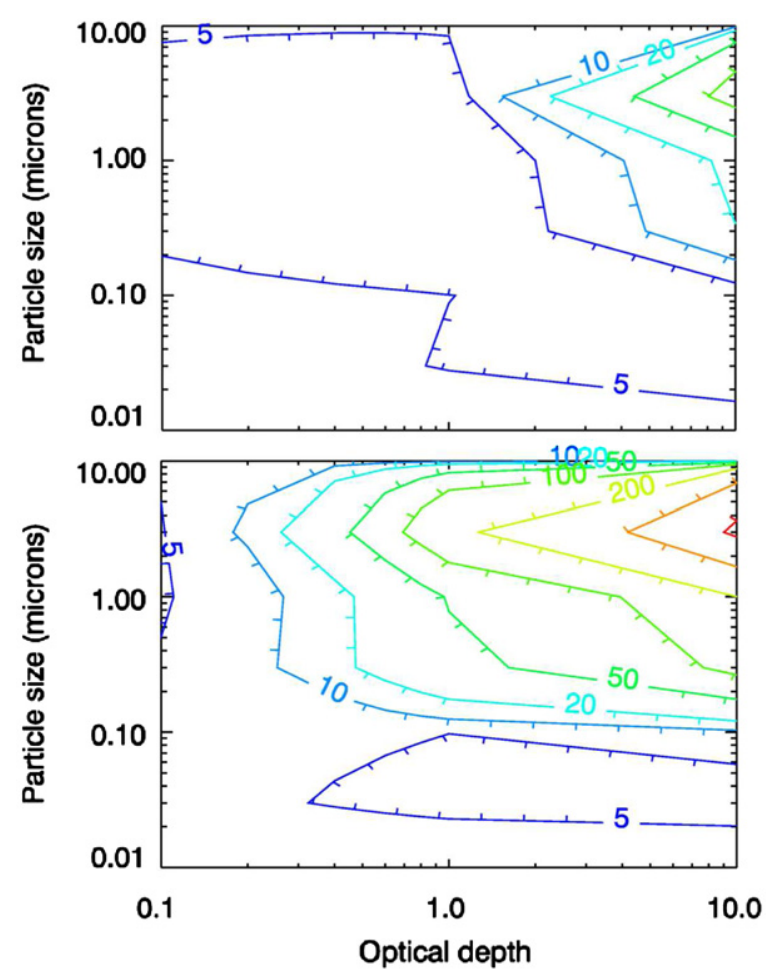

Figure 5. $\chi^{2}$ contour plots for $100 \times$ solar Na and a cloud from 1000-100 mbar (top) and 100-10 mbar (bottom). The best-fitting models for the first case occur for an optical depth of 1 and a particle size between 0.3 and $3 \mu \mathrm{m}$; for the second case, the best-fit model occurs for an optical depth of 10 and a particle size of $0.03 \mu \mathrm{m}$.

(A color version of this figure is available in the online journal.)

pressure range, and models where the cloud particles are uniformly distributed throughout the atmosphere as, for many cases, we can obtain a good fit using either (Figure 3). Based on the results of Parmentier et al. (2013), a uniform cloud of particles up to $0.1 \mu \mathrm{m}$ in size is plausible; we may expect a cloud formed of larger particles to be located lower in the atmosphere due to sedimentation and rain out. For particle sizes of up to $0.1 \mu \mathrm{m}$, the uniform cloud model contains fewer assumptions (as we do not define a cloud base or cloud top pressure) and Occam's razor suggests that this should be preferred for any given values of $\mathrm{Na} V \mathrm{VMR}$, particle radius, and optical depth, where the quality of fit is equal.

Heng \& Demory (2013) also compare a set of cloud models to the HST/STIS spectrum and find similar degeneracy between $\mathrm{Na}$ abundance and the cloud. They find that the abundance of $\mathrm{Na}$ relative to the abundance of cloud particles decreases by a factor $10^{5}$ if the cloud particle size decreases by a factor 10 from $100 \mathrm{~nm}$ to $10 \mathrm{~nm}$. For sub- $\mu \mathrm{m}$ particles, we find a similar result. The best fit $\mathrm{Na} \mathrm{VMR}$, and therefore the number of $\mathrm{Na}$ atoms, is roughly constant as a function of particle size if optical depth and cloud altitude are fixed (Figure 3 ), and we require a factor $10^{5}$ increase in the number of cloud particles as the size decreases from $100 \mathrm{~nm}$ to $10 \mathrm{~nm}$ to maintain a fixed optical depth. As in the work of Heng \& Demory (2013), we find that the shape of the reflection spectrum is largely dictated by the particle size and the Na VMR.

\section{1. $\mathrm{K}, \mathrm{TiO}$, and $\mathrm{VO}$}

So far, we have only examined the effect of $\mathrm{Na}$ on the visible spectrum and have ignored other species. We include $\mathrm{K}$ in the model atmosphere $(0.1 \mathrm{ppmv})$, and Figure 6 shows the effect of

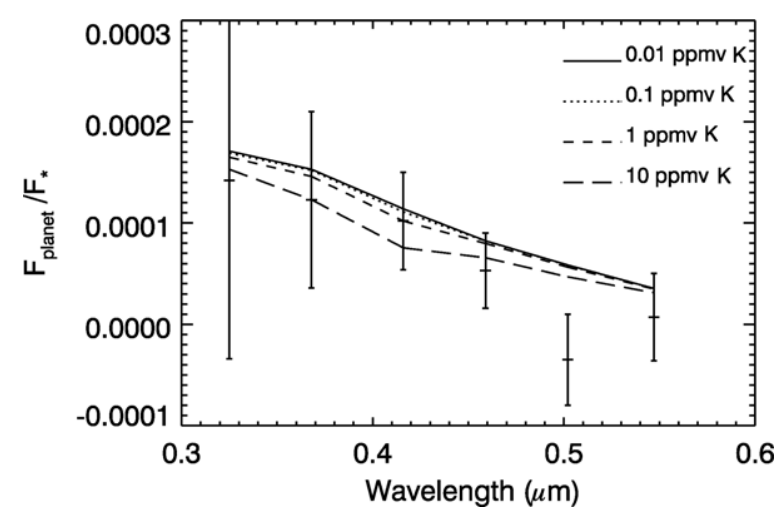

Figure 6. Synthetic, cloud-free STIS spectra containing different abundances of $\mathrm{K}$. Varying the amount of $\mathrm{K}$ in the model does not significantly affect the spectrum.

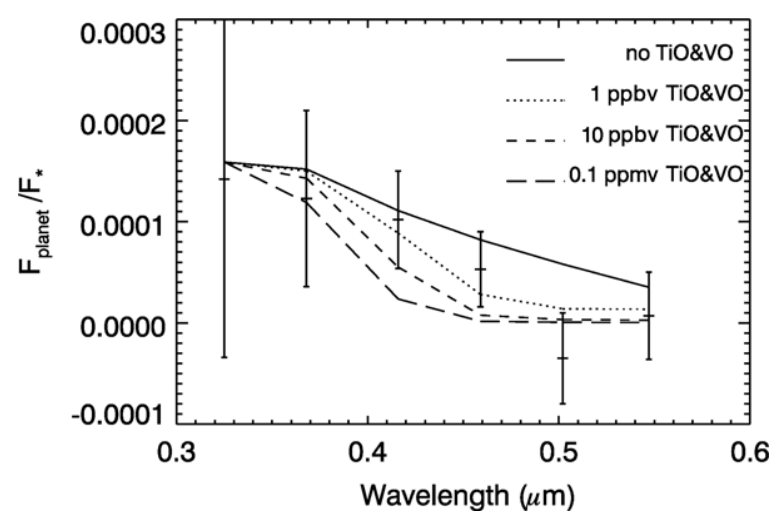

Figure 7. Synthetic, cloud-free STIS spectra including TiO and VO. The presence of $\mathrm{TiO}$ and $\mathrm{VO}$ is unlikely in $\mathrm{HD} 189733 \mathrm{~b}$ as it is expected to be too cold for $\mathrm{TiO}$ and $\mathrm{VO}$ condensates to evaporate, but including these species in the model produces a good fit to the spectrum.

varying the abundance over 4 orders of magnitude for a cloudfree model. It can be seen that $\mathrm{K}$ has a much smaller effect on the shape of the spectrum than $\mathrm{Na}$ (Figure 2), and the magnitude of the change in flux ratio is smaller than the error bars on the STIS spectrum, justifying our decision not to vary K in our main analysis.

HD $189733 \mathrm{~b}$ is not expected to have a hot enough photosphere for absorption due to gaseous $\mathrm{TiO}$ and $\mathrm{VO}$ to be present in spectra (Fortney et al. 2010), and no features due to these gases have been observed in the transmission spectrum; however, it is still worthwhile testing the effect of these gases on the spectrum should they unexpectedly be present. We show a series of cloud-free synthetic spectra including absorption due to $\mathrm{TiO}$ and VO (using absorption data from R. Freedman 2011, private communication) in Figure 7. The effect is much more significant than the effect of varying the $\mathrm{K}$ abundance, and including some $\mathrm{TiO}$ and $\mathrm{VO}$ as well as $\mathrm{Na}(10 \mathrm{ppb})$ improves the spectral fit from the case without $\mathrm{TiO}$ and $\mathrm{VO}$. This should not be taken as evidence that $\mathrm{TiO}$ and $\mathrm{VO}$ are present on HD 189733b, as the problem is degenerate and we still expect this planet to be too cold for these species to be present in the gas phase; in addition, $\mathrm{TiO}$ and VO have not yet been detected in the atmospheres of hotter planets (e.g., Sing et al. 2013), so the presence of these molecules in planetary atmospheres is questionable.

\subsection{Cloud Composition}

We have so far assumed that any cloud on HD $189733 \mathrm{~b}$ is made of enstatite. Morley et al. (2012) propose a range of other 

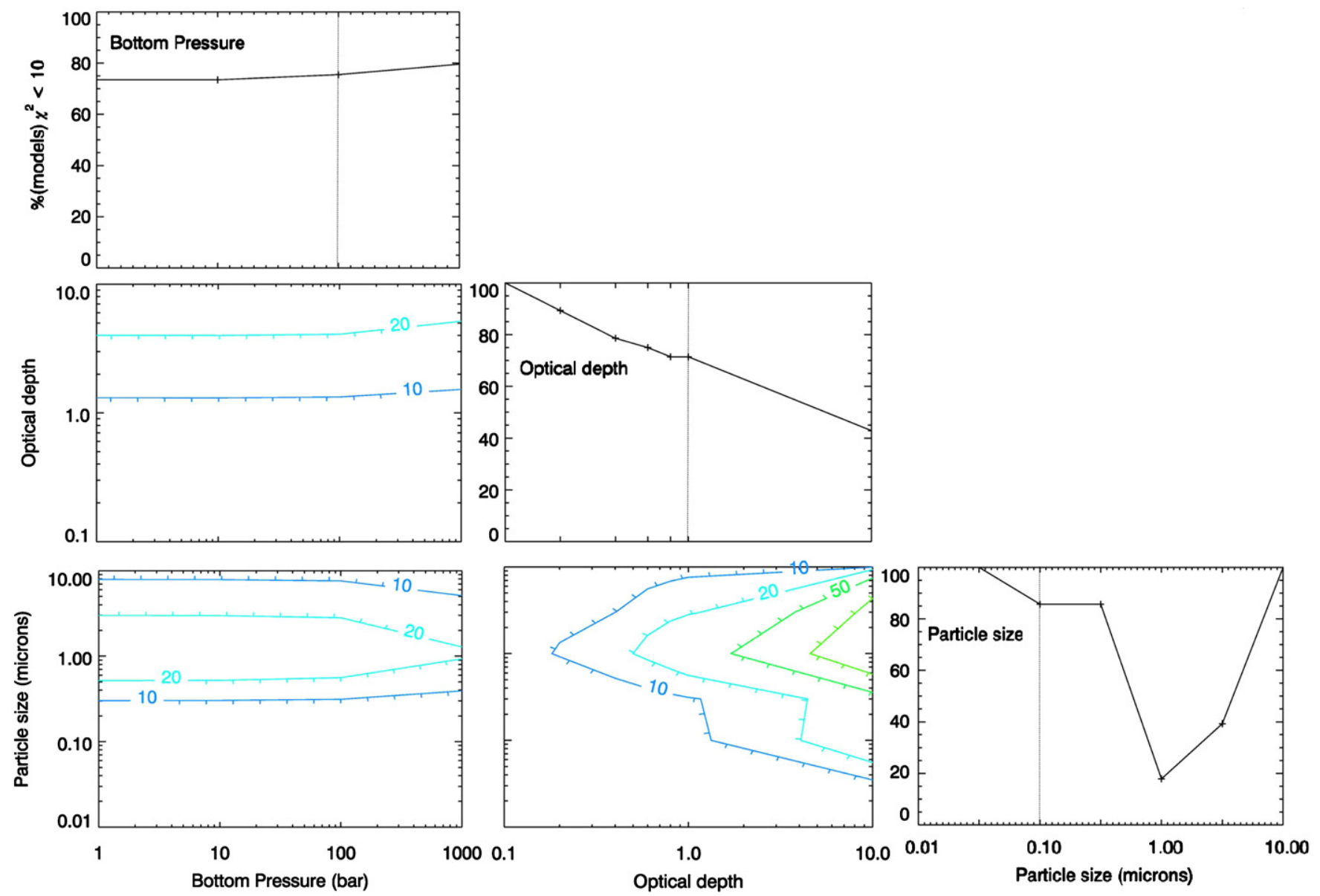

Figure 8. Contour plots of $\chi^{2}$ goodness-of-fit parameter for cloud bottom pressure, optical depth and particle size for MnS clouds. Also plotted are the percentages of models with $\chi^{2}$ of $<10$ for each parameter. The dashed lines indicate the values used for each parameter when held fixed.

(A color version of this figure is available in the online journal.)

minerals that are likely to form clouds on brown dwarfs, and $\mathrm{MnS}$ is a possible candidate for brown dwarfs with similar temperatures to HD 189733b. We repeat the analysis for a sodium VMR of 5 ppmv, with clouds composed of MnS. The results are presented in Figure 8.

It is clear that the particle size/optical depth parameter space still encompasses the most important spectral variability for the cloud, as discussed in the Appendix of Lee et al. (2013a). However, choosing $\mathrm{MnS}$ instead of enstatite results in a greater range of cloud models that produce a fit to the data with $\chi^{2}<10$, but no models provide a very good fit to the data $\left(\chi^{2}<5\right)$. This is due to the fact that MnS has a different refractive index to enstatite, which affects the scattering efficiency and phase function of the particles and serves to illustrate the complexity of the cloud parameter space.

\subsection{Comparison with Terminator Cloud Models}

The results presented here illustrate the highly degenerate nature of any cloudy atmosphere solutions for HD 189733b. If we are to assume that the dayside atmosphere is similar to the terminator atmosphere, then we can place some further constraint on the cloud by comparing to results from the transmission spectrum. The analysis of Lee et al. (2013b) shows that the best-fit models for the terminator atmosphere, for a uniformly distributed cloud, have particles $\sim 0.1 \mu \mathrm{m}$ in size, with an optical depth of 0.01 at $1.0 \mu \mathrm{m}$. This corresponds to our set of solutions for uniformly distributed $0.1 \mu \mathrm{m}$ particles with an optical depth of 0.5 at $0.25 \mu \mathrm{m}$, as the extinction crosssection of $0.1 \mu \mathrm{m}$ enstatite particles is a factor $\sim 50$ smaller at $1.0 \mu \mathrm{m}$ than at $0.25 \mu \mathrm{m}$. A $\chi^{2}$ of $<6$ is achieved for these particles if the Na VMR is 5 ppmv, and $\chi^{2}<3$ for a VMR of $50 \mathrm{ppmv}$. If we can expect the cloud structure to be the same at the terminator and on the dayside, we could therefore place some limits on the Na VMR.

Whether or not we expect the same cloud to form is a question for general circulation models of HD $189733 \mathrm{~b}$ to solve. So far, few have dealt with cloudy atmospheres, with some exceptions. Parmentier et al. (2013) present a 3D circulation model for the somewhat hotter planet HD 209458b, including passive tracer particles of various sizes to examine the relative effects of circulation and sedimentation on aerosols and condensates; they find that, whereas larger particles sediment out and show significant longitudinal variability in abundance, the number of $0.1 \mu \mathrm{m}$-sized tracer particles remains relatively uniform with longitude. This indicates that a cloud composed of $0.1 \mu \mathrm{m}$ particles could easily be approximately uniform throughout the atmosphere of a hot Jupiter; and therefore, we may be observing the same cloud deck on the dayside as Lee et al. (2013b) observe at the terminator, although it is likely that different altitudes are probed.

\section{THERMAL EMISSION RETRIEVAL}

We now take a subset of the cloud models used in Section 3 and examine their effect on retrievals of temperature and 


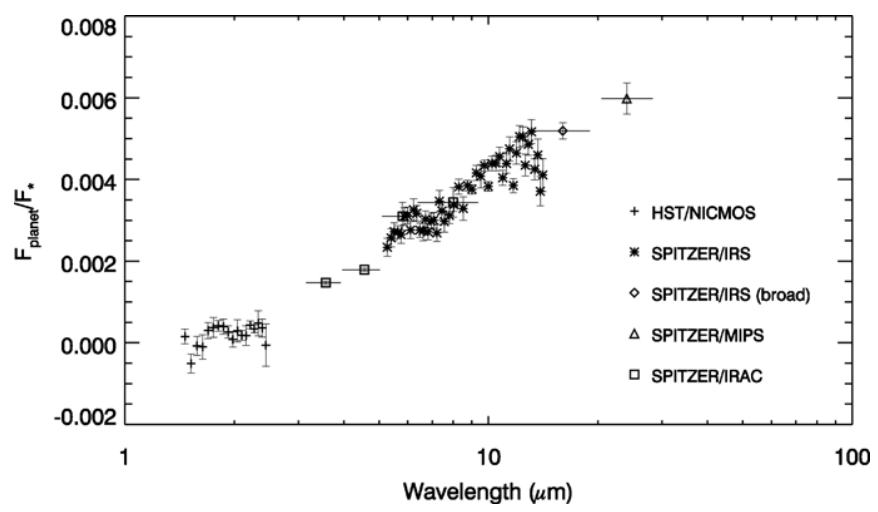

Figure 9. Data for the thermal emission retrieval. NICMOS points are as reanalyzed in this work; $3.6 \mu \mathrm{m}, 4.5 \mu \mathrm{m}$, and $8 \mu \mathrm{m}$ IRAC points are from Knutson et al. (2012); the IRS spectrum is from Grillmair et al. (2008); the IRS broadband point was originally measured by Deming et al. (2006); 5.8 and $8.0 \mu \mathrm{m}$ Spitzer/IRAC and MIPS points are as measured by Charbonneau et al. (2008), along with a reanalysis of the broadband IRS point.

molecular abundances from the infrared secondary eclipse spectrum (Figure 9). The retrieval is performed as in Lee et al. (2012) using hemispheric integration for non-scattering runs and a $45^{\circ}$ emission angle approximation for scattering runs. We also use our reanalyzed NICMOS spectrum and the warm Spitzer 3.6, 4.5, and $8 \mu \mathrm{m}$ points presented in Knutson et al. (2012). We include the cloud model in the model atmosphere but do not make any attempt to retrieve it; instead, we examine its effect on the other parameters in the retrieval.

Firstly, we repeat the cloud-free analysis of Lee et al. (2012) with the addition of the reanalyzed NICMOS data points; Lee et al. (2012) state that the NICMOS spectrum provides information about the temperature profile in the deep atmosphere and also the abundances of trace gases, and as these data have changed, we expect to retrieve a different temperature profile and abundances. We perform the retrieval of temperature as a function of pressure, over 50 atmospheric levels with a correlation length of 1.5 in $\ln$ (pressure), and altitude-independent abundances of $\mathrm{H}_{2} \mathrm{O}, \mathrm{CO}_{2}, \mathrm{CO}$, and $\mathrm{CH}_{4}$ using a range of different temperature and gas abundance priors; since the problem is underconstrained, it is necessary to test the influence of the prior on the retrieved property. We present the results of this analysis for temperature in Figure 10 and Table 4. The best-fit retrieved temperature profile (Figure 10 top left) and gas abundances are averaged over all runs. The temperature sensitivity weighting function averaged over all wavelengths is shown qualitatively in Figure 10 by a shaded bar, with the peak (lightest color) occurring between 1 and 100 mbar; this corresponds to the region of the atmosphere probed by the majority of the data, and therefore, the temperature is least dependent on the temperature prior in this region; this is shown in Figure 10 top right, in which the retrieved profiles for different priors converge between 1 and 100 mbar.

The temperature structure is most sensitive to the Knutson et al. (2012) Spitzer/IRAC 3.6 and $4.5 \mu \mathrm{m}$ points as these data have by far the smallest fractional error, meaning that they drive the temperature retrieval. This accounts for the differences in the shape of the retrieved T-p profile compared with that of Lee et al. (2012). The $3.6 \mu \mathrm{m}$ weighting function peaks at around 1 mbar and constrains the stratospheric temperature, whereas the $4.5 \mu \mathrm{m}$ weighting function peaks at the tropopause, at around 100 mbar.

In Figure 10 it can be seen that the greatest degeneracy between temperature and gas abundance occurs for $\mathrm{H}_{2} \mathrm{O}$ and
Table 4

The Mean Retrieved Values for each Gas VMR, and the Minimum and Maximum Retrieved Values over all Priors

\begin{tabular}{lclcc}
\hline \hline & Mean $\pm \sigma \times 10^{-4}$ & Min & Max & Lee et al. \\
\hline $\mathrm{H}_{2} \mathrm{O}$ & $11 \pm 11$ & 1.9 & 46 & 6.7 \\
$\mathrm{CO}_{2}$ & $22 \pm 28$ & 1.3 & 96 & 20 \\
$\mathrm{CO}$ & $2.1 \pm 4.1$ & 0.010 & 22 & 29 \\
$\mathrm{CH}_{4}$ & $0.26 \pm 0.09$ & 0.01 & 0.42 & 0.0019 \\
\hline
\end{tabular}

Notes. $\mathrm{H}_{2} \mathrm{O}$ and $\mathrm{CH}_{4}$ can be reasonably well constrained, but $\mathrm{CO}_{2}$ and $\mathrm{CO}$ have $\sigma$ larger than $100 \%$ of the mean value. The best-fit values for Lee et al. (2012) are given for comparison.

$\mathrm{CO}_{2}$, with the precise shape of the retrieved T-p profile between 1 and 100 mbar varying as a function of the $\mathrm{H}_{2} \mathrm{O}$ and $\mathrm{CO}_{2}$ abundance priors. This is because the peak of the temperature weighting function and therefore the pressure probed varies with atmospheric opacity, and these two gases are the most spectrally active. The increase in the error bars for the NICMOS spectrum means that the gas abundance retrievals are more sensitive to the prior than found by Lee et al. (2012); so for a small $\mathrm{H}_{2} \mathrm{O}$ or $\mathrm{CO}_{2}$ prior abundance the retrieved abundance is smaller, the atmospheric opacity is less, and the pressure that was probed is higher. The reverse is true for high prior abundances of $\mathrm{H}_{2} \mathrm{O}$ or $\mathrm{CO}_{2}$. The effects of changing the $\mathrm{CO}$ and $\mathrm{CH}_{4}$ abundances are smaller, but similar.

The average temperature profile that was retrieved is largely similar to that of Line et al. (2014), except that we see a slight decrease in temperature above the 1 mbar level compared with Line et al. (2014). This is probably due to the fact that we retrieve temperature for each atmospheric level individually, with an assumed correlation length (1.5 in log-pressure coordinates), whereas Line et al. (2014) parameterize the temperature profile. Therefore, the upper atmosphere temperature retrieved by Line et al. (2014) cannot vary freely with respect to the temperature between 1 and 100 mbar, where the sensitivity is greatest, whilst we retrieve a continuous profile.

The mean, standard deviation and range of retrieved gas VMRs are given in Table 4. The standard deviations are highest for $\mathrm{CO}_{2}$ and $\mathrm{CO}$, implying a high dependence on the prior. The retrieved values for $\mathrm{H}_{2} \mathrm{O}$ and $\mathrm{CO}_{2}$ are similar to that of Lee et al. (2012) but the value for $\mathrm{CO}$ is smaller; although, due to the dependence on the prior, this result must be viewed with caution. However, we retrieve a much higher $\mathrm{CH}_{4}$ abundance than Lee et al. (2012), roughly a factor of 10 higher than the Spitzer-derived upper limit of Madhusudhan \& Seager (2009). Our higher value for $\mathrm{CH}_{4}$ is similar to the result of Line et al. (2014), and so it is likely to be a result of the revised 3.6 and $4.5 \mu \mathrm{m}$ points which are also used by these authors.

We now seek to investigate the effect of the cloud on the retrieved temperature profile and gas abundances. We use the average temperature prior and a priori gas VMRs of $10^{-4}$ for all retrieved species. The parameters for the set of models we use are shown in Table 5. These correspond to some of the best-fitting solutions from the previous analysis that have very different infrared optical depths, so they are likely to affect the thermal emission retrieval differently.

We show the retrieved temperature profiles and gas VMRs for each of the cloud models, as well as the gas abundances for the cloud-free case (Figure 11, Table 6). We perform each of the retrievals twice, first using the fast, extinction-only disk integration technique as in Lee et al. (2012), and secondly using multiple scattering as in Section 3, except we approximate the 

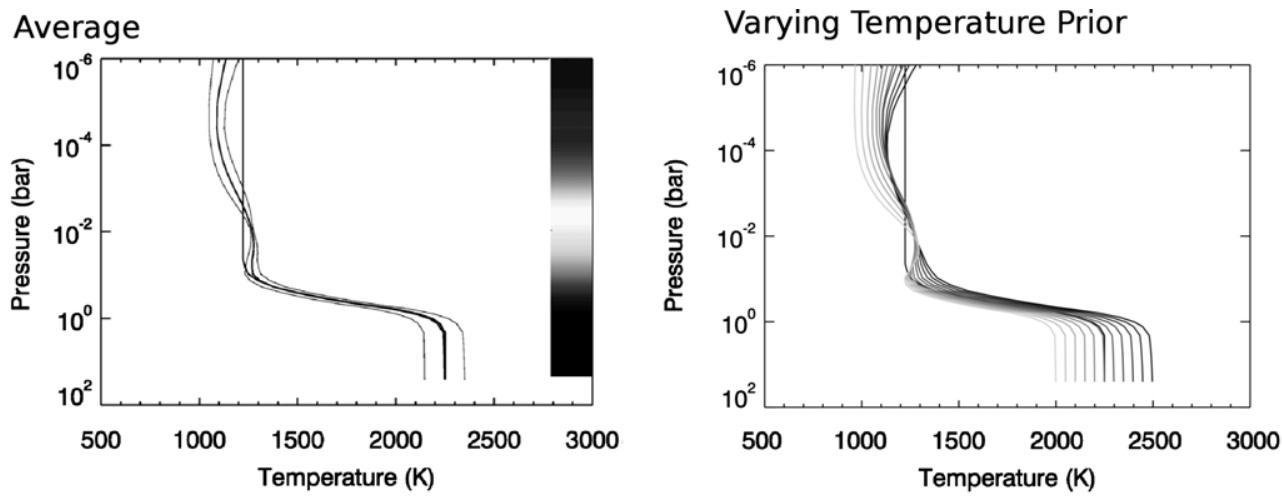

Varying $\mathrm{H} 2 \mathrm{O}$ Prior

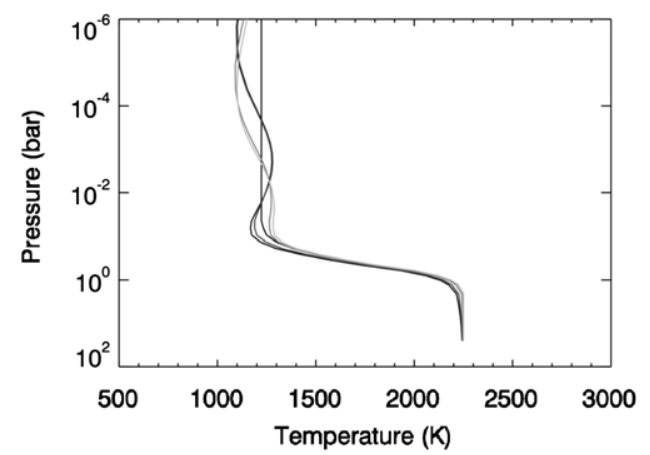

Varying CO2 Prior

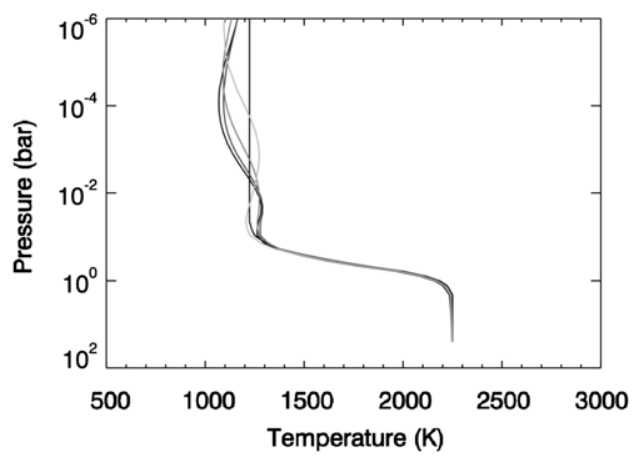

Varying CO Prior

Varying $\mathrm{CH} 4$ Prior
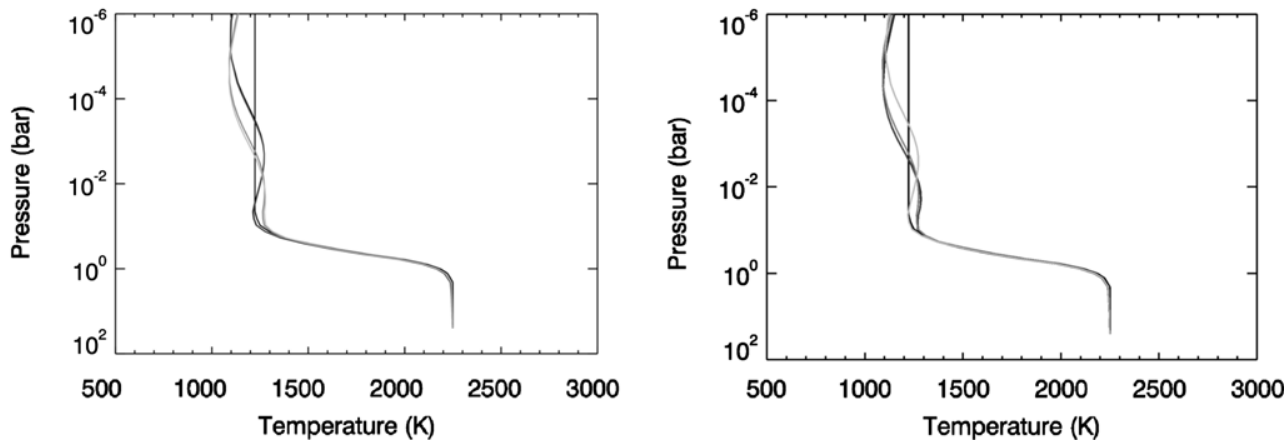

Figure 10. Retrieved temperature profiles for a range of different priors. The mean and standard deviation are shown in the top left; the shaded bar shows the average sensitivity to temperature at each altitude, across all wavelengths, with black being low sensitivity and white high sensitivity. The maximum sensitivity occurs between 1 and 100 mbar. The varying temperature prior is the best-fit case from Lee (2012), shifted at $50 \mathrm{~K}$ intervals from $-250 \mathrm{~K}$ (lightest gray line) to $+250 \mathrm{~K}$ (darkest gray line), with a prior error of $\pm 50 \mathrm{~K}$. We also vary the prior altitude-independent gas $\mathrm{VMR}$ for $\mathrm{H}_{2} \mathrm{O}, \mathrm{CO}_{2}, \mathrm{CO}$, and $\mathrm{CH}_{4}$. The darkest-lightest gray colors correspond to priors for each gas of $1 \mathrm{ppmv}, 10 \mathrm{ppmv}, 100 \mathrm{ppmv}$, and $1000 \mathrm{ppmv}$. It can be seen that the greatest degeneracy occurs for $\mathrm{CO}_{2}$. The temperature prior used where the gas priors are varying is the thick black line, with a prior error of $\pm 50 \mathrm{~K}$.

Table 5

The Parameters for the Cloud Models Included in Thermal Emission Retrievals, to Test the Dependence on Cloud Properties

\begin{tabular}{lccc}
\hline \hline Model & Base $\mathrm{p}$ & $0.25 \mu \mathrm{m}$ O. D. & Particle Radius \\
\hline 1 & Uniform & 10 & $0.1 \mu \mathrm{m}$ \\
2 & $1000 \mathrm{mbar}$ & 1 & $0.01 \mu \mathrm{m}$ \\
3 & $1000 \mathrm{mbar}$ & 1 & $3 \mu \mathrm{m}$ \\
4 & $10 \mathrm{mbar}$ & 10 & $0.03 \mu \mathrm{m}$ \\
\hline
\end{tabular}

disk with a single $45^{\circ}$ emission/incidence angle as the five-angle calculation is extremely time-consuming for a full retrieval.

It is clear from Figure 11 and Table 6 that the thermal emission retrieval is largely insensitive to the inclusion of clouds corresponding to our best-fitting models. This is due to the fact that the majority of best-fitting models with optical depths greater than 1 at $0.25 \mu \mathrm{m}$ have small particle sizes, and therefore the extinction drops rapidly as a function of wavelength. These particles have little effect at wavelengths longer than $1 \mu \mathrm{m}$ because the particle size is small with respect to the wavelength of the light, and so the optical depth in the infrared is negligible even though it is significant at shorter wavelengths. This means that even higher optical depths of $0.1 \mu \mathrm{m}$-sized particles would be unlikely to change the temperature retrieval.

The exception is the retrieved $\mathrm{H}_{2} \mathrm{O}$ and $\mathrm{CO}_{2}$ abundances and temperature profile for cloud model 4 , using an extinction-only assumption; including a high (10-1 mbar) cloud of $0.03 \mu \mathrm{m}$ sized particles results in a larger retrieved $\mathrm{CO}_{2}$ abundance, which in turn affects the shape of the temperature profile in this pressure range (as in Figure 10). Where solutions are degenerate, as in this case, it is possible for small changes in one model variable (here, the cloud optical depth) to result in different best-fit solutions being obtained. In Figure 12, we show the synthetic spectrum for the model 4 retrieved case and the spectrum for 

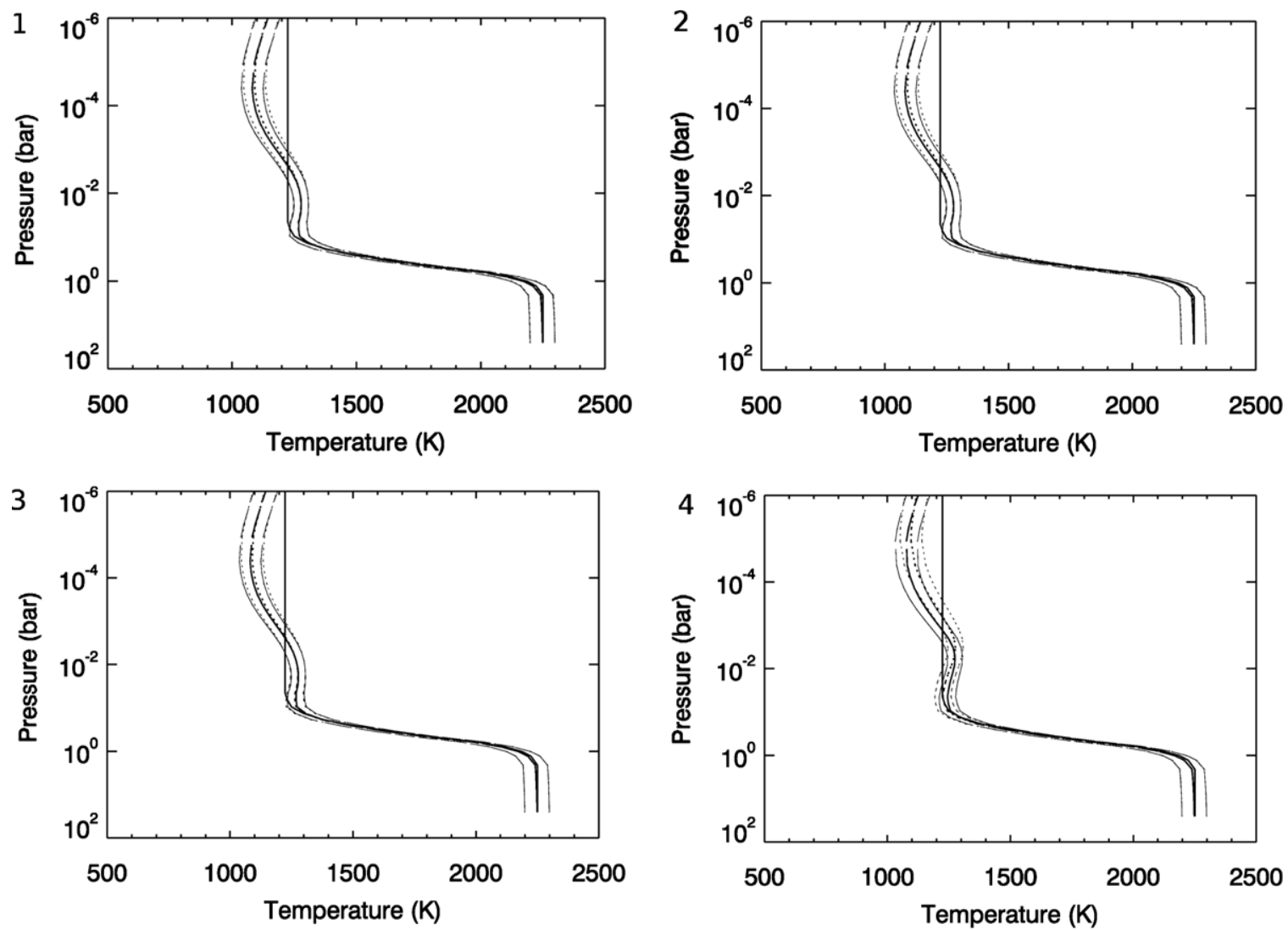

Figure 11. Retrieved temperature profiles for each cloud model. Thick solid lines show the a priori temperature; dotted lines show the extinction-only retrieval; and dashed lines show the multiple scattering retrieval. Thick lines show the retrieved value, and thin lines show the error envelope.

Table 6

The Retrieved Gas VMRs for each of the Four Cloud Models, Including the Cloud-free Case (Model 5), for Extinction-only (left) and Multiple Scattering (right)

\begin{tabular}{lcccc}
\hline \hline Model & $\mathrm{H}_{2} \mathrm{O}$ VMR $\left(10^{-4}\right)$ & $\mathrm{CO}_{2}$ VMR $\left(10^{-4}\right)$ & $\mathrm{CO}$ VMR $\left(10^{-4}\right)$ & $\mathrm{CH}_{4} \mathrm{VMR}^{-4}\left(0^{-4}\right)$ \\
\hline 1 & $9.7 \pm 4.9 / 11 \pm 6$ & $8.9 \pm 4.6 / 8.1 \pm 4.2$ & $1.4 \pm 1.4 / 1.4 \pm 1.4$ & $0.25 \pm 0.11 / 0.26 \pm 0.11$ \\
2 & $9.6 \pm 4.9 / 11 \pm 6$ & $9.0 \pm 4.7 / 8.2 \pm 4.3$ & $1.4 \pm 1.4 / 1.4 \pm 1.4$ & $0.25 \pm 0.11 / 0.26 \pm 0.11$ \\
3 & $8.4 \pm 4.4 / 11 \pm 5$ & $8.1 \pm 4.2 / 7.8 \pm 4.1$ & $1.4 \pm 1.4 / 1.4 \pm 1.4$ & $0.24 \pm 0.10 / 0.25 \pm 0.11$ \\
4 & $3.8 \pm 2.0 / 6.8 \pm 3.7$ & $31 \pm 17 / 13 \pm 7$ & $1.3 \pm 1.3 / 1.3 \pm 1.3$ & $0.31 \pm 0.09 / 0.31 \pm 0.11$ \\
5 & $9.7 \pm 4.9$ & $8.9 \pm 4.6$ & $1.4 \pm 1.4$ & $0.25 \pm 0.11$ \\
\hline
\end{tabular}

Notes. The cloud-free case was modeled using an extinction-only approximation.

a forward model with a retrieved temperature profile and gas abundances as for a cloud-free model but instead including a model 4 cloud. The spectra are very similar, with $\chi^{2}$ of 138 and 142 , respectively, demonstrating that the difference in retrieved $\mathrm{CO}_{2}$ abundance for this case should not be treated as significant. Additionally, the multiple scattering retrieval for model 4 yields a lower abundance of $\mathrm{CO}_{2}$ and a temperature profile more similar to those retrieved using other cloud models. For the case of HD 189733b, therefore, it seems that an accurate retrieval of temperature and atmospheric composition from the thermal emission spectrum may not be critically dependent on an understanding of the cloud properties.

\section{DISCUSSION}

We have used a very simple set of cloud models to investigate the acceptable parameter space for the HD 189733b STIS spectrum. The quality of the data at this stage do not warrant more detailed modeling, but it is instructive to consider the

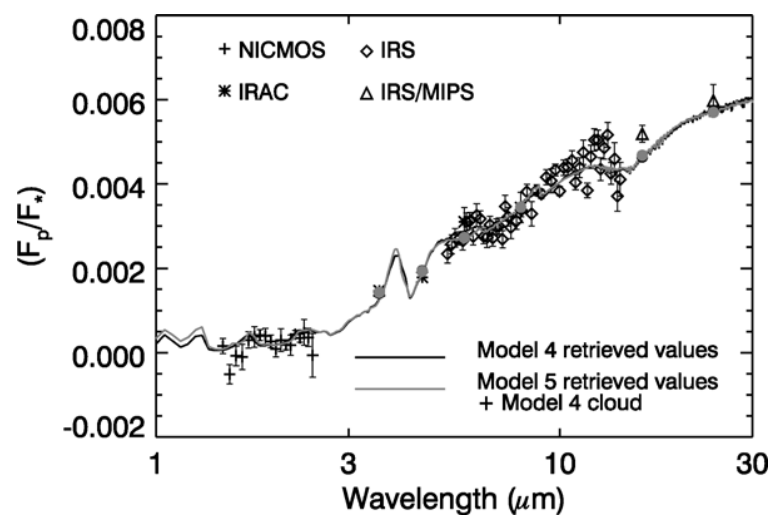

Figure 12. We show synthetic spectral fits for a forward model containing the cloud from model 4 but the retrieved parameters from model 5 (gray) and the retrieved parameters and cloud from model 4 (black). The difference between the two spectra is small, with $\chi^{2}$ of 142 and 138, respectively. The numbers of spectral points (71) and degrees of freedom (54) are the same for both cases. The circles show the high-resolution synthetic spectra convolved at the Spitzer IRAC, broadband IRS, and MIPS bandpasses. 
most important steps that could be taken to improve this model in anticipation of future results.

\subsection{Monodisperse Particles}

We have assumed that all the particles in the cloud are of a single size. This is of course not likely to be the case in practice; cloud models based on microphysical processes indicate that particle sizes will change as a function of altitude, and at a given altitude particle size distributions may be relatively broad, for example, as discussed by Helling et al. (2008). Coalescence processes, condensational growth, and sedimentation will result in the presence of larger grains toward the bottom of the cloud deck.

Broadening the size distribution will make the cloud optical depth more uniform as a function of wavelength; extinction due to cloud particles is most efficient when the particle size is comparable to the wavelength of light. This may result in the cloud having a larger effect on the thermal emission spectrum than we find in this work; although in most cases, we find that particle sizes of larger than $0.1 \mu \mathrm{m}$ do not provide a good fit to the data at short wavelengths, this does not preclude larger particles being present as they would scatter the short wavelength light less strongly than smaller particles.

When the data are available that would allow us to test a more complex model, we consider that investigating different particle size distributions would be the most important step to take, as particle size is the property that has the largest effect on the spectrum.

\subsection{Particle Composition}

The composition of clouds on cool brown dwarfs and hot exoplanets is treated somewhat differently in different models. Morley et al. (2012) consider a range of possible cloud compositions for specific temperatures, under the assumption that if clouds of more than one species are present they will form at different temperatures and, therefore, altitudes. Helling et al. (2008) have a very different model, in which multiple species condense onto the same $\mathrm{TiO}_{2}$ "seed particle" (Helling $\&$ Woitke 2006), thus forming grains of mixed composition. This significantly complicates the calculation of spectral properties for these clouds; however, Helling et al. (2008) find that, for a low-gravity atmosphere with $T_{\text {eff }} \sim 1300 \mathrm{~K}$, the enstatite is the constituent with the largest effect on the spectrum, meaning that enstatite probably represents the best approximation to the multi-component grains.

We tested the effect of including $\mathrm{MnS}$, as proposed by Morley et al. (2012) for cool brown dwarfs, and we found that the different spectral properties of the material do affect the spectrum but to a lesser extent than the size of the particles. Therefore, particle composition is an interesting route for further investigation but is of secondary importance to developing more detailed representations of particle size.

\subsection{Effect of Temperature Profile}

We have modeled the STIS reflection spectrum using the bestfit temperature profile from Lee (2012), but we then retrieved a different profile using the thermal emission spectrum. We tested the effect on the best-fit cloud properties of including the altered temperature profile in our analysis of the cloud. We found that the changes to the spectral shape were negligible compared to the error bars on the STIS spectrum (Figure 13), and the $\chi^{2}$ values for different cloud models do not change

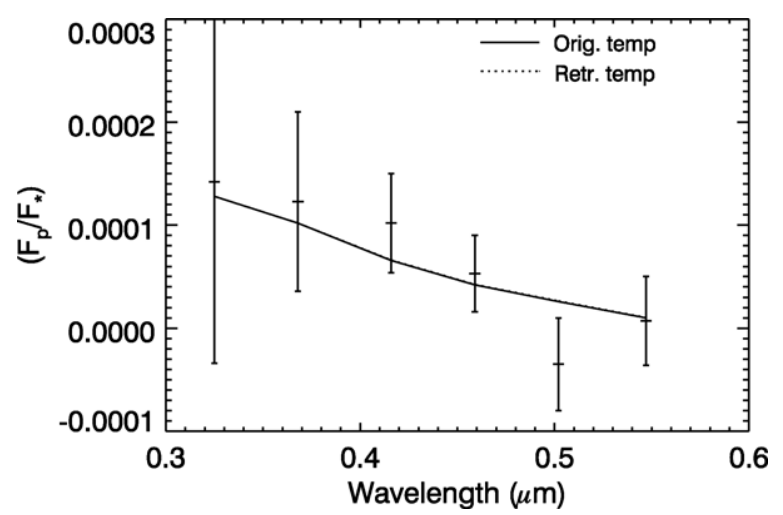

Figure 13. We show two STIS spectrum models, both containing the model 1 cloud properties. One is the model generated using the original temperature profile; the other uses the retrieved temperature profile for model 1 . The synthetic spectra are indistinguishable from each other.

greatly. Therefore, small inaccuracies in the temperature profile we use would not significantly affect our conclusions; the same cloud models provide good/poor fits to the measured spectrum regardless of the temperature profile that was used.

The reason for the limited effect of temperature is likely to be that the absorption due to $\mathrm{Na}$ at the altitudes to which we are sensitive is dominated by pressure-broadened line wings. Temperature variation is more likely to affect the line core absorption at higher altitudes but at the resolution of the STIS spectrum such variation would not be seen. The STIS measurements also lie in a wavelength region where the signal is dominated by reflected starlight, rather than light emitted from the planet.

\section{CONCLUSION}

We find that a large range of enstatite cloud models can fit the measured HST/STIS spectrum. Cloud-free model atmospheres are also acceptable solutions, although we consider this to be less plausible due to the clear evidence from the transmission spectrum that HD 189733b is cloudy (Pont et al. 2013; Lee et al. 2013b) and the likelihood that small particles are evenly distributed in hot Jupiter atmospheres (Parmentier et al. 2013). Small enstatite particles $(=<0.1 \mu \mathrm{m})$ and $50 \mathrm{ppmv}$ of $\mathrm{Na}$ provide the best fit to the STIS data of the examples we test; we find an overlap with the models of Lee et al. (2013b) for a uniformly distributed $0.1 \mu \mathrm{m}$ cloud with an optical depth of 0.5 at $0.25 \mu \mathrm{m}$. However, the problem is extremely degenerate, and we cannot exclude solutions with larger cloud particles.

The retrieval of temperature and atmospheric composition from the thermal emission spectrum is relatively insensitive to the inclusion of clouds in the model atmosphere for our bestfitting models. This suggests that for the case of HD $189733 \mathrm{~b}$ accurate retrieval of temperature and gaseous abundances from the thermal emission spectrum is possible, even without detailed knowledge of the cloud properties. Solution degeneracy prevents firm conclusions from being drawn about the nature of the cloud on HD 189733b; the current quality and coverage of spectroscopic data for most exoplanets is therefore insufficient to simultaneously constrain temperature structure, gaseous abundances, and multiple cloud properties. Given the additional complexity (and therefore number of degenerate solutions) introduced to the retrieval problem when clouds are included, this implies that the best approach with the currently available secondary eclipse data is to use cloud-free model atmospheres 
for temperature retrieval. As data quality improves, alternative, more detailed cloud models must be explored, and their effect on the emission spectrum must be reassessed.

J.K.B. acknowledges the support of the John Fell Oxford University Press (OUP) Research Fund for this research and L.N.F. is supported by a Royal Society Research Fellowship. We thank the anonymous reviewer for his/her report, and Tom Evans, Frédéric Pont, David Sing and Kevin Heng for helpful discussions about the visible albedo observations.

\section{REFERENCES}

Baines, E. K., McAlister, H. A., ten Brummelaar, T. A., et al. 2008, ApJ, 680,728

Barstow, J. K., Aigrain, S., Irwin, P. G. J., Fletcher, L. N., \& Lee, J.-M. 2013a, MNRAS, 434, 2616

Barstow, J. K., Aigrain, S., Irwin, P. G. J., et al. 2013b, MNRAS, 430, 1188

Barstow, J. K., Tsang, C. C. C., Wilson, C. F., et al. 2012, Icar, 217, 542

Borysow, A. 2002, A\&A, 390, 779

Borysow, A., \& Frommhold, L. 1989, ApJ, 341, 549

Borysow, A., \& Frommhold, L. 1990, ApJL, 348, L41

Borysow, A., Frommhold, L., \& Moraldi, M. 1989, ApJ, 336, 495

Borysow, A., Jorgensen, U. G., \& Zheng, C. 1997, A\&A, 324, 185

Bouchy, F., Udry, S., Mayor, M., et al. 2005, A\&A, 444, L15

Charbonneau, D., Knutson, H. A., Barman, T., et al. 2008, ApJ, 686, 1341

de Wit, J., Gillon, M., Demory, B.-O., \& Seager, S. 2012, A\&A, 548, A128

Deming, D., Harrington, J., Seager, S., \& Richardson, L. J. 2006, ApJ, 644, 560

Deming, D., Wilkins, A., McCullough, P., et al. 2013, ApJ, 774, 95

Evans, T. M., Pont, F., Sing, D. K., et al. 2013, ApJL, 772, L16

Fortney, J. J., Shabram, M., Showman, A. P., et al. 2010, ApJ, 709, 1396

Gibson, N. P., Aigrain, S., Roberts, S., et al. 2012, MNRAS, 419, 2683

Gibson, N. P., Pont, F., \& Aigrain, S. 2011, MNRAS, 411, 2199

Goody, R. M., \& Yung, Y. L. 1989, Atmospheric Radiation: Theoretical Basis (New York: Oxford Univ. Press)

Grillmair, C. J., Burrows, A., Charbonneau, D., et al. 2008, Natur, 456, 767

Heiter, U., Barklem, P., Fossati, L., et al. 2008, JPhCS, 130, 012011

Helling, C., \& Woitke, P. 2006, A\&A, 455, 325

Helling, C., Woitke, P., \& Thi, W.-F. 2008, A\&A, 485, 547
Heng, K., \& Demory, B.-O. 2013, ApJ, 777, 100

Henyey, L. G., \& Greenstein, J. L. 1941, ApJ, 93, 70

Huffman, D. R., \& Wild, R. L. 1967, PhR, 156, 989

Huitson, C. M., Sing, D. K., Vidal-Madjar, A., et al. 2012, MNRAS, 422, 2477

Irwin, P. G. J., Teanby, N. A., \& Davis, G. R. 2009, Icar, 203, 287

Irwin, P. G. J., Teanby, N. A., de Kok, R., et al. 2008, JQSRT, 109, 1136

Knutson, H. A., Lewis, N., Fortney, J. J., et al. 2012, ApJ, 754, 22

Lacis, A. A., \& Oinas, V. 1991, JGR, 96, 9027

Lecavelier Des Etangs, A., Pont, F., Vidal-Madjar, A., \& Sing, D. 2008, A\&A, 481, L83

Lee, J.-M. 2012, PhD thesis, Univ. of Oxford

Lee, J.-M., Fletcher, L. N., \& Irwin, P. G. J. 2012, MNRAS, 420, 170

Lee, J.-M., Heng, K., \& Irwin, P. G. J. 2013a, ApJ, 778, 97

Lee, J.-M., Irwin, P. G. J., Fletcher, L. N., Heng, K., \& Barstow, J. K. 2013b, arXiv: 1310.5868

Line, M. R., Knutson, H., Wolf, A., \& Yung, Y. 2014, ApJ, 783, 70

Line, M. R., Zhang, X., Vasisht, G., et al. 2012, ApJ, 749, 93

Madhusudhan, N., \& Seager, S. 2009, ApJ, 707, 24

Mandell, A. M., Drake Deming, L., Blake, G. A., et al. 2011, ApJ, 728, 18

Morley, C. V., Fortney, J. J., Marley, M. S., et al. 2012, ApJ, 756, 172

Parmentier, V., Showman, A. P., \& Lian, Y. 2013, A\&A, 558, 91

Plass, G. N., Kattawar, G. W., \& Catchings, F. E. 1973, ApOpt, 12, 314

Pont, F., Sing, D. K., Gibson, N. P., et al. 2013, MNRAS, 432, 2917

Redfield, S., Endl, M., Cochran, W. D., \& Koesterke, L. 2008, ApJL, 673, L87

Rodgers, C. D. 2000, Inverse Methods for Atmospheric Sounding (Singapore: World Scientific)

Rothman, L. S., Gordon, I. E., Barber, R. J., et al. 2010, JQSRT, 111, 2139

Rothman, L. S., Wattson, R. B., Gamache, R., Schroeder, J. W., \& McCann, A. 1995, Proc. SPIE, 2471, 105

Scott, A., \& Duley, W. W. 1996, ApJS, 105, 401

Sing, D. K., Lecavelier des Etangs, A., Fortney, J. J., et al. 2013, MNRAS, 436, 2956

Southworth, J. 2008, MNRAS, 386, 1644

Sudarsky, D., Burrows, A., \& Pinto, P. 2000, ApJ, 538, 885

Swain, M. R., Vasisht, G., Tinetti, G., et al. 2009, ApJL, 690, L114

Tashkun, S. A., Perevalov, V. I., Teffo, J.-L., Bykov, A. D., \& Lavrentieva, N. N. 2003, JQSRT, 82, 165

Tinetti, G., Deroo, P., Swain, M. R., et al. 2010, ApJL, 712, L139

Waldmann, I. P., Tinetti, G., Drossart, P., et al. 2012, ApJ, 744, 35

Wenger, C., \& Champion, J. P. 1998, JQSRT, 59, 471

Winn, J. N., Johnson, J. A., Marcy, G. W., et al. 2006, ApJL, 653, L69 